\title{
An Automated Visual Inspection System for the Classification of the Phases of Ti-6Al-4V Titanium Alloy
}

\author{
Antonino Ducato, Livan Fratini, Marco La Cascia, and Giuseppe Mazzola \\ DICGIM - Dipartimento di Ingegneria Chimica Gestionale Informatica e Meccanica \\ Università degli Studi di Palermo, Viale delle Scienze, bd 6, 90128 Palermo, Italy \\ \{antonino.ducato, livan.fratini, marco.lacascia, \\ giuseppe.mazzola\}@unipa.it
}

\begin{abstract}
Metallography is the science of studying the physical properties of metal microstructures, by means of microscopes. While traditional approaches involve the direct observation of the acquired images by human experts, Computer Vision techniques may help experts in the analysis of the inspected materials. In this paper we present an automated system to classify the phases of a Titanium alloy, Ti-6Al-4V. Our system has been tested to analyze the final products of a Friction Stir Welding process, to study the states of the microstructures of the welded material.
\end{abstract}

Keywords: Titanium, Ti-6Al-4V, Metallography, Computer Vision, Automated Visual Inspection, SVM, Texture.

\section{Introduction and Previous Works}

In an a industrial workflow, visual inspection and quality control of the manufacturing process, until to the end product, are traditionally performed by human experts. Even if usually the human expertise works better than a machine application, it is much slower and more expensive. Moreover, in certain applications human inspection is tedious (repetitive actions) or dangerous (e.g. underwater inspection, nuclear or chemical industry, etc.). Computer vision is an effective solution in such cases [1].

In this work we focused on a specific field of the industrial engineering: metallography, that is the study of the physical properties of metals, by optical and electron microscopy. One of the aims of metallography is to study the microstructures of an inspected metal, under certain working conditions. According to metallography, structures which are coarse enough to be discernible by the naked eye or under low magnifications are termed macrostructures, while those which require high magnification to be visible are termed microstructures. Even if useful information can often be gained by examination with the naked eye of the surface of metal objects, microscopes are required for the examination of the microstructure of the metals. Optical microscopes are used for resolutions down to roughly the wavelength of light (about half a micron) and electron microscopes are used for details below this level, down to atomic resolution. Particular features of interest are: grain size, phases content, distribution of 
phases, elongated structures formed by plastic deformation. The examination of materials by optical microscopy is essential in order to understand the relationship between properties and microstructure. While the traditional approach involved the direct observation of the acquired images by human experts, resulting in a qualitative analysis of the results, with the diffusion of digital image processing techniques the analysis process became faster, simpler and more precise. Computer Vision techniques have been used in Metallography Image Analysis[2,3] to study the properties of sinteredsteel and the nickel-based superalloy [4], for the automated classification of heat resistant steel structures[5], for segmenting the phases of high strength low alloy steel [6], to study the pit formation on a titanium alloy [7], and for the segmentation of a of a two-phase Ti-6Al-2Mo-2Cr-Fe titanium alloy[8]. Tejrzanowski et al. [9] presented a review of different techniques for the estimation of the size, shape and spatial distribution of structural elements of engineering materials.

In our work we proposed an automated inspection system to study the properties of a titanium alloy, and in particular of its microstructures, in order to classify the parts of the inspected material into different mechanical-physical phases. The paper is organized as follow: section 2 describes the features of the titanium alloy and one of its most important welding techniques; section 3 presents our classification system; section 4 discuss our experimental results; a conclusive section ends the paper.

\section{Titanium Properties}

Today, titanium alloys are common, readily available engineered metals that compete directly with stainless and specialty steels, nickel-based alloys and composites. In addition to its attractive high strength characteristics for aerospace use, titanium's exceptional corrosion resistance, derived from its protective oxide film, has encouraged extensive application in seawater, marine and industrial chemical service over the past fifty years. Today, titanium and its alloys are extensively used for: aircraft engines and airframes, spacecraft, chemical and petrochemical production, power generation, nuclear waste storage, navy ship components, automotive components, food and pharmaceutical processing, medical implants and surgical devices. An important aspect of this kind of alloy is its microstructural evolution as function of the thermo-mechanical history, depending on the applied manufacturing process. It is possible to differentiate three different kind of microstructures, called phases, having different mechanical and physical properties: Alpha, Beta, and Alpha+Beta (fig. 1). The formation and the behavior of each phase is linked to the addition of alloying elements (called stabilizers) to titanium, which enables physical-chemical effects on the creation of the single microstructural type. The most commonly used titanium alloy is Ti-6Al-4V, that is the object of study of our work. It has a chemical composition of $6 \%$ aluminum, $4 \%$ vanadium, $0.25 \%$ (maximum) iron, $0.2 \%$ (maximum) oxygen, and the remainder titanium. These alloy elements make its microstructure composed, at room temperature, of $91 \%$ of Alpha phase and $9 \%$ of Beta phase (Fig. 2). It is significantly stronger than commercially pure titanium while having similar thermal properties. Among its many advantages, it is heat treatable[11]. 

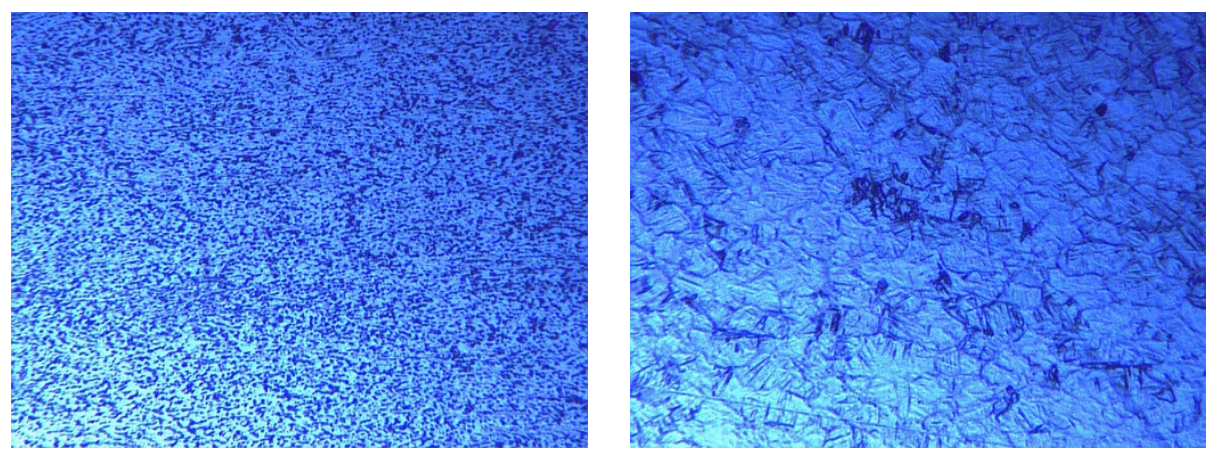

Fig. 1. Metallography of Alpha (light) and Beta (dark) and Alpha+Beta microstructure types, on the left and on the right respectively

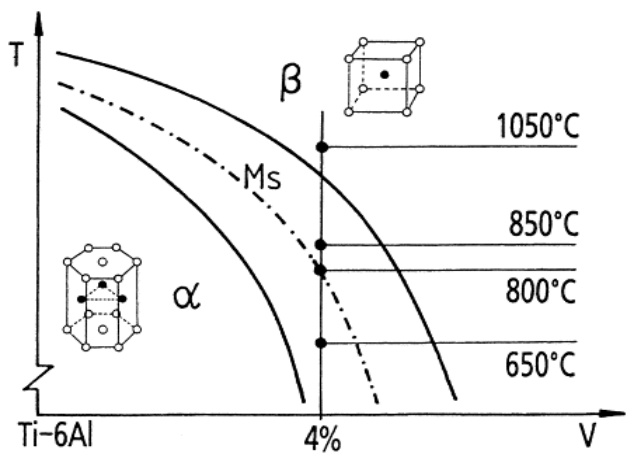

Fig. 2. Ti-6Al-4V phase diagram

\subsection{Titanium Alloys Friction Stir Welded Joints}

Friction Stir Welding is an important new non-fusion technique for joining sheet and plate material [12]. FSW was invented by TWI (The Welding Institute) in 1991, and is a TWI licensed technology. The tool is rotated and plunged into the material so that the shoulder works on the plate surface and the probe is buried in the workpiece. The friction between the rotating tool and the plate material generates heat, and the high normal pressure from the tool causes a plasticized zone to form around the probe. The tool is then traversed, frictionally heating and plasticizing new material as it moves along the joint line [13]. Although the majority of common titanium alloys are generally weldable by conventional means, problems with workpiece distortion, and poor weld quality, can occur. The development of FSW offers the possibility of a new method of producing high quality, low distortion, welds in Ti sheet and plates .

The parent material was found to consist of a rolled microstructure of elongated gains of alpha (light) in a matrix of alpha and beta (dark). In the deformed weld zone, the microstructure shows evidence of Alpha-to-Beta phase transformation. Significant 
grain growth appears to occur at this elevated temperature, producing large equiaxed beta grains in the weld center. The beta phase reverts on cooling, and the resultant weld microstructure consists of large alpha grains with a smaller amount of retained beta. The weld root zone microstructure in this case shows that only partial transformation has occurred in this region. After FSW, the preparation of a specimen to reveal the microstructure of the welded material involves the following steps: sawing the section to be examined, mounting in resins, coarse grinding, grinding on progressively finer emery paper, polishing using alumina powder or diamond paste on rotating wheel, etching in dilute acid, washing in Alcohol and drying. The specimen is then ready to be inspected by microscope.

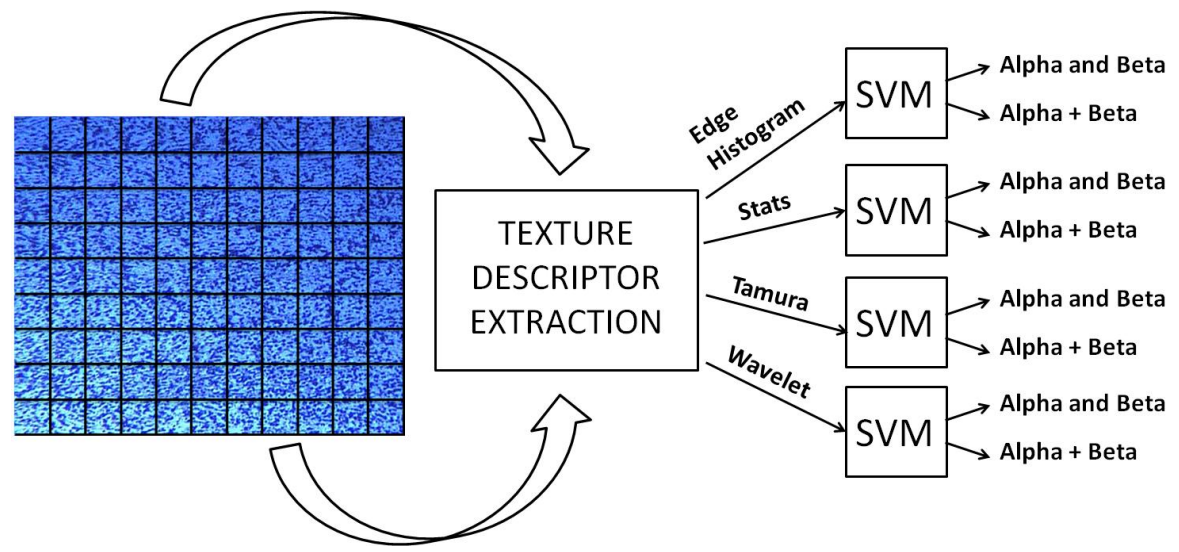

Fig. 3. Overall scheme of the proposed system

\section{Proposed System}

The main goal of our work is to find a compact and functional description of the image information, in order to classify all the areas of the image into the two possible classes: "Alpha and Beta" and "Alpha+Beta". In this paper we compare the results obtained with several low level features descriptors, using a common testing framework. The scheme of the overall system is shown in fig.3:

- Images (size $\mathrm{M} \times \mathrm{N})$ are first decomposed into a grid of $(\mathrm{m} \times \mathrm{n})$ non overlapping subblocks of size $B \times B$, where $m=M / B$ and $n=N / B$. The value of $B$ will be further described in the experimental section.

- Features are extracted from each sub-block and concatenated to form a $\mathrm{BxBxNi}$ vector, where $\mathrm{Ni}$ is the size of a single block descriptor vector. In our work we analyzed a set of texture descriptors, which are briefly described in the next section. Color information, in this case, is useless as the images in the dataset are, in practice, monochrome. 
- Feature vectors are then used to train a Support Vector Machine used as binary classifier. SVM is the most used and the simplest solution whenever a binary classification problem has to be solved, therefore is well suited for our goals. Information about the SVM setup will be given in the experimental section. Each feature is used to train separately a classifier.

- After training, we test the classifiers to evaluate the precision of the system.

If a block is classified as "Alpha and Beta" class, it is further processed to discriminate between Alpha and Beta phases, and to assign to each pixel another label. In this case a simple adaptive threshold method is applied. For each block all the pixels whose grey values are above the average value of the block are labeled as Alpha (lightest areas), and the other ones as Beta (darkest areas). Two constraints (a minimum and maximum value) are imposed to this threshold value, to treat also the rare case in which all the pixels of a block are of the same class (i.e. all dark or all light). We preferred to use an adaptive threshold approach, rather than a global one, as it works also in case of not uniform illumination during the acquisition of the image.

\subsection{Texture Descriptors}

Texture is one of the most studied image features in Computer Vision, Image Processing and Computer Graphics applications. It can be considered as a measure of the perceived image surface variations. For our purpose we tested 4 different standard texture descriptors:

- Statistical: mean, standard deviation, skewness and kurtosis of the pixel grey values. Output is a 4-dimensional vector.

- Wavelet: the sub-band energy of the coefficients of a 2-level wavelet transform of the image, as described in [14]. Output is a 7-dimensional feature vector.

- Tamura [15] descriptors: Contrast, Coarseness and Directionality properties from the Tamura set of features. Output is a 3-dimensional feature vector.

- Edge Histogram [16]: in our simplified version, we filter blocks with 4 directional (vertical, horizontal, 45, 135) and a non-directional Roberts-like operators. Mean and standard deviations of the filtered blocks are considered as descriptors. Output is a 10-dimensional feature vector.

\section{Experimental Results}

Our dataset is composed by more than 150 images, of size $1079 \times 816$. In our experiments, for simplicity of annotation, we selected 10 examples of images representing the "pure" Alpha and Beta phase and 10 representing the "pure" Alpha+Beta phase. We divided each image into blocks of size $\mathrm{BxB}$. We tried with different values of $\mathrm{B}$ but, at last, we decided to set $\mathrm{B}=80$, as it is the nearest value to the size of a microstructure in an Alpha+Beta phase. Each image is then divided into a grid of $13 \times 10$ 


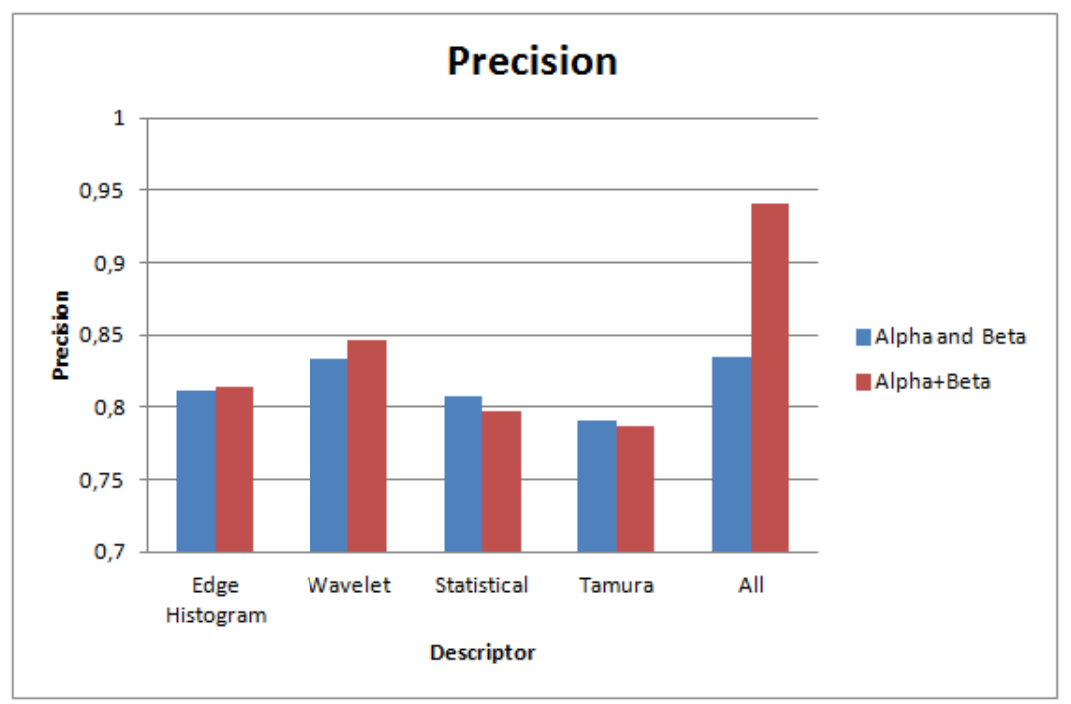

Fig. 4. Experimental results, with the single descriptors and with a combination of them

blocks, (residual pixels on the borders of the image are discarded). As a result, we had 1300 Alpha and Beta and 1300 Alpha+Beta samples. In our tests, our dataset was randomly split into training and testing images, equally. Each block is classified separately, as in the general case, Alpha and Beta and Alpha+Beta areas may be present in the same specimen. We measured the accuracy of the classifier, trained with the different descriptor vectors, in terms of precision, for both the classes. Each experiment has been repeated 100 times, and results averaged. Figure 4 shows the results obtained with the different texture descriptors Our experiments have been also repeated, using different kernels and parameters for the SVM classifier. We achieved our best results with a RBF kernel, and with $\mathrm{C}=1$ and $\gamma=1$. Results are shown only for the best configuration. We have obtained around $80 \%$ precision with all descriptors, and almost $85 \%$ with the Wavelet descriptor. We furthermore concatenated the descriptors into a single vector, trained another classifier, and repeated the tests. We measured a higher value of precision ("All" bar in fig. 4), especially for the Alpha+Beta phase (almost 95\%). The result is that the combination of all the texture descriptors is more effective, than the single ones, in the representing the Alpha+Beta microstructures.

A numerical evaluation of the Alpha/Beta segmentation algorithm, in case of Alpha and Beta images, is very difficult to give as, due to the resolution with which the images were acquired, there is not a precise and clear separation between Alpha and Beta pixels into the images. According to a subjective evaluation, the results are satisfactory and the values of percentage of the two phases in the Alpha and Beta specimens is consistent with the expected results. Figure 5 show a visual example of the output of our classifier, for an Alpha and Beta case.

In terms of efficiency, for the single step of descriptor extraction, we obtained similar results. Time to extract information from an input image is less than a second for all the analyzed descriptors. 

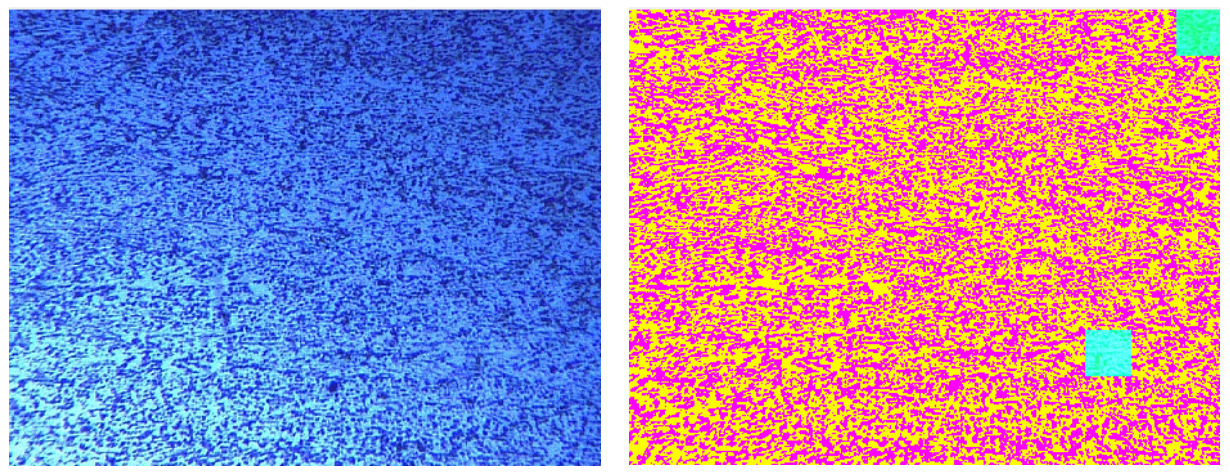

Fig. 5. A visual example. An image of Alpha and Beta input specimen (left) and the output classification (right), using the Tamura descriptor. Cyan areas have been (wrongly) classified as Alpha+Beta. Yellow pixels are classified as Alpha and purple pixels as Beta.

\section{Conclusions}

Nowadays the use of computer aided software allows to design a great number of manufacturing processes both from a pure mechanical and structural point of view. The actual race consists on finding a computer aided system taking into account the technological knowledge about a particular alloy in order to obtain a FEM (Finite Element Method) suite able to predict, as function of thermo-mechanical history dictated by the considered process, both the classical thermo-mechanical and the metallurgical response of the material. The state of art of the numerical codes is ready to offer several solution to improve a numerical model containing all the requested knowledge and mathematical formulation to simulate and carry-out good (after a correct calibration) results in terms of phase evolution predictions. However these types of numerical models need for a proper set-up, based on a direct comparison with the experimental data provided by the considered real process study cases, with the aim of verifying if the parameters and the used mathematical laws are correct. Unfortunately the comparison between experimental and numerical results is very difficult especially when the user have to evaluate, by observing a metallography, the phase content to obtain an accurate quantitative information. It often happens that it is not possible to obtain a direct link between the output data of the numerical code and the experimental observation of the phenomena. Therefore a computer software for image analysis is needed in order to obtain a numerical evaluation of typology of phases and their percentage content respect to the observed area. This numerical tool should be the connecting ring able to create a CAE (Computer Aided Engineering) system for the design of forming processes of the considered alloy. With this goal, we developed our classification system, that can be a very helpful and powerful instrument to support metal science experts in the analysis process. Moreover, future versions of our system would be able to detect also imperfections and defects (grooves, holes) of the welded materials. 


\section{References}

1. Malamas, E.N., Petrakis, E.G.M., Zervakis, M., Petit, L., Legat, J.D.: A survey on industrial vision systems, applications and tools. Image and Vision Computing 21(2), 171-188 (2003)

2. Tan, W., Chengdong, C., Zhao, C., Li, S.: Study on key technology of metallographical image processing and recognition. In: Control and Decision Conference, CCDC 2008, pp. 1832-1837. Chinese (July 2008)

3. Gegner, J., Öchsner, A.: Digital image analysis in quantitative metallography. Pract. Metallogr. 38(9), 499-513 (2001)

4. Komenda, J.: Automatic recognition of complex microstructures using the Image Classifier. Materials Characterization 46(2-3), 87-92 (2001)

5. Topalova, I., Mihailov, A., Tzokev, A.: Automated classification of heat resistant steel structures based on neural networks. In: IEEE 25th Convention of Electrical and Electronics Engineers in Israel, IEEEI 2008, December 3-5, pp. 437-440 (2008)

6. Chatterjee, O., Das, K., Dutta, S., Datta, S., Saha, S.K.: Phase extraction and boundary removal in dual phase steel micrographs. In: 2010 Annual IEEE India Conference (INDICON), December 17-19, pp. 1-5 (2010)

7. Codaro, E.N., Nakazato, R.Z., Horovistiz, A.L., Ribeiro, L.M.F., Ribeiro, R.B., Hein, L.R.O.: An image analysis study of pit formation on Ti-6Al-4V. Materials Science and Engineering: A 341(1-2), 202-210 (2003)

8. Chrapoński, J., Szkliniarz, W.: Quantitative metallography of two-phase titanium alloys. Materials Characterization 46(2-3), 149-154 (2001)

9. Tejrzanowski, T., Spychalski, W., Rozniatowski, K.K.: Image Based Analysis of Complex Microstructures of Engineering Materials. Int. J. Appl. Math. Comput. Sci. 18(1), 33-39 (2008)

10. Sha, W., Malinov, S.: Titanium Alloy: Modelling of Microstructure, Properties and Applications. Woodhead Publishing Series in Metals and Surface Engineering, vol. 31 (April 2009)

11. Bruschi, S., Poggio, S., Quadrini, F., Tata, M.E.: Workability of Ti-6Al-4V alloy at high temperatures and strain rates. Materials Letters 58(27-28), 3622-3629 (2004)

12. Buffa, G., Ducato, A., Fratini, L.: Numerical procedure for residual stresses prediction in friction stir welding. Finite Elements in Analysis and Design 47(4), 470-476 (2011)

13. Fratini, L., Buffa, G., Shivpuri, R.: Mechanical and metallurgical effects of in process cooling during friction stir welding of AA7075-T6 butt joints. Acta Materialia 58(6), 2056-2067 (2010)

14. Serrano, N., Savakis, A., Luo, J.: Improved scene classification using efficient low-level features and semantic cues. Pattern Recognition 37, 1773-1784 (2004)

15. Tamura, H., Mori, S., Yamawaki, T.: Texture features corresponding to visual perception. IEEE Trans. Syst. Man Cybern. 8(6), 460-473 (1978)

16. Won, C.S., Park, D.K.: Efficient Use of MPEG-7 Edge Histogram Descriptor. ETRI Journal 24(1), 23-30 (2002) 